\title{
Lab on a Tip: Atomic Force Microscopy as a Versatile Analytical Tool for Nano-bioscience
}

\author{
Evan Angelo Quimada Mondarte, ${ }^{1}$ Hiroyuki Tahara, ${ }^{1}$ Kasinan Suthiwanich, ${ }^{1}$ \\ Subin Song, ${ }^{1}$ Fan Wang, ${ }^{1}$ and Tomohiro Hayashi ${ }^{1,2^{*}}$ \\ ${ }^{1}$ Tokyo Institute of Technology, Department of Materials Science and Engineering, School of Materials and \\ Chemical Technology, 4259 Nagatsuta-cho, Midori-ku, Yokohama, Kanagawa 226-8502, Japan \\ ${ }^{2}$ JST-PRESTO, 4-1-8 Hon-cho, Kawaguchi, Saitama 332-0012, Japan
}

(Received September 3, 2020; accepted December 3, 2020)

Keywords: scanning probe microscopy, atomic force microscopy, single-molecule force spectroscopy, surface force

Scanning probe microscopy (SPM) is a powerful method for visualizing the structure of materials at the nanoscale. In particular, atomic force microscopy (AFM) has become one of the most used analytical tools in various fields such as physics, chemistry, and biology. Here, we introduce representative works in nano-bioscience. First, we look back on the history of SPM and introduce the application of AFM in this field. Next, we review surface force and singlemolecule force measurements, which unveiled molecular processes at biointerfaces. Surface force measurements revealed the mechanism underlying the macroscopically observed protein and cell resistance of artificial monolayers and biomolecules. Meanwhile, single-molecule force spectroscopy has enabled researchers to explore the complex interaction of biomolecules from a microscopic viewpoint. These findings will contribute not only to the fundamental understanding of biomolecular processes but also to the design of new nano-biodevices.

\section{Introduction}

The history of scanning probe microscopy (SPM) started in the field of solid-state surface science. Scanning tunneling microscopy (STM) enabled the first-ever imaging of atomic structures of conductive solid materials in 1982. ${ }^{(1)}$ The invention of atomic force microscopy (AFM) in 1987 made it possible to observe surface structures of insulators, resulting in the drastic expansion of research targets. ${ }^{(2)}$ Also, AFM measurements can be performed in various environments in liquids and gases as well as in a vacuum, spurring the application of AFM in biology. ${ }^{(3,4)}$ Researchers became able to investigate biomolecules and cells under a physiological condition, and imaging with AFM unveiled the functions of biomolecules, such as molecular recognition, enzymic reactions, and self-assembly in a native state. ${ }^{(5,6)}$

In addition to imaging, AFM can also be used for measurements of a force acting in water by monitoring the deflection of a cantilever. By employing a cantilever with an appropriate spring constant and surface modification, researchers can measure the interaction between the

*Corresponding author: e-mail: tomo@mac.titech.ac.jp

https://doi.org/10.18494/SAM.2021.3069 
tip and substrate in water with pico-Newton sensitivity. ${ }^{(3)}$ Interactions in water are responsible for many biological phenomena, including molecular recognition between ligands and receptors, aggregation, enzymic reactions, and adsorption. To understand the mechanisms behind these molecular processes, AFM has often been used to measure surface forces and intermolecular interactions in water.

\section{Surface Force Analysis in Water}

\subsection{Interactions in water: DLVO and non-DLVO forces}

In nano-bioscience, the environment is usually water. The interaction between objects in water has often been interpreted in the context of DLVO theory, in which the interaction is described with an electrostatic double layer and van der Waals forces. DLVO theory has succeeded in explaining the interaction between inorganic materials such as mica under various solution conditions. ${ }^{(7)}$ In contrast with inorganic materials, organic materials, particularly soft materials and biomolecules, which are hydrated and possess flexible structures, exhibit different interactions in water. In addition to the DLVO forces, the contribution from non-DLVO forces such as steric repulsion (sometimes referred to as the entropic or volume-exclusion force) and water-mediated forces (hydrophobic and hydrophilic interactions) is significant. ${ }^{(8)}$

In particular, non-DLVO forces are difficult to predict because theories to describe them quantitatively have not been established so far. In this sense, the precise measurement of nonDLVO forces is necessary. Surface force apparatus (SFA) is the most powerful technique for measuring the force acting between solid surfaces with the precise control of distances. ${ }^{(9)}$ The disadvantage of SFA is the limitation of the measurable interfaces, because users need to construct all surfaces on mica. In contrast, surface force measurements by AFM allow us to investigate various surfaces. ${ }^{(10-12)}$ We often employ colloidal probes in AFM measurements, for which a silica or polystyrene colloid with a diameter of several to several tens of $\mu \mathrm{m}$ [Fig. 1(a)] is fixed at the end of the cantilever. This technique enables us to measure surface forces quantitatively with high sensitivity because of the well-defined geometry and large diameter of colloidal probes. In addition, various surface modifications (evaporation, polymer coating, chemical conjugation) are possible for both the probe and substrate, providing a versatile platform for the measurements.

\subsection{Investigation of water-mediated forces by force measurements using AFM}

Water-mediated forces have been considered to play essential roles in interfacial phenomena such as friction, adsorption, adhesion, and corrosion. ${ }^{(13)}$ In nano-bioscience, experimental and theoretical works proved that water-mediated forces are driving forces for the self-assembly of molecules and the suppression of non-specific interactions in molecular recognition events. ${ }^{(14)}$ Readers may have noticed that water-mediated forces can be both attractive and repulsive. The Debye length at physiological salt concentration is about $0.7 \mathrm{~nm}$. Therefore, the importance of water-mediated forces can be high in biological processes. 


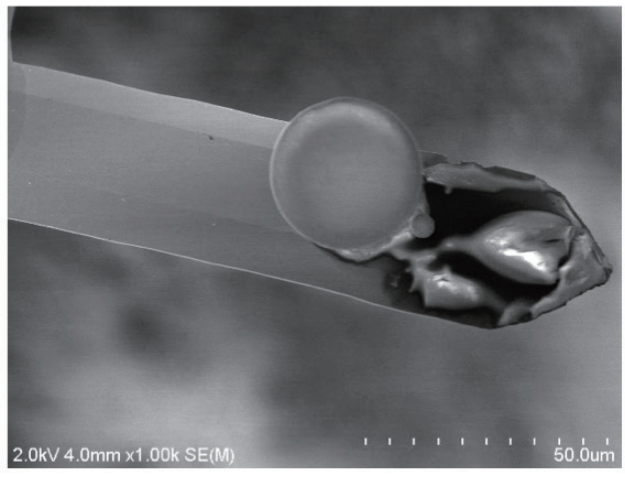

(a)

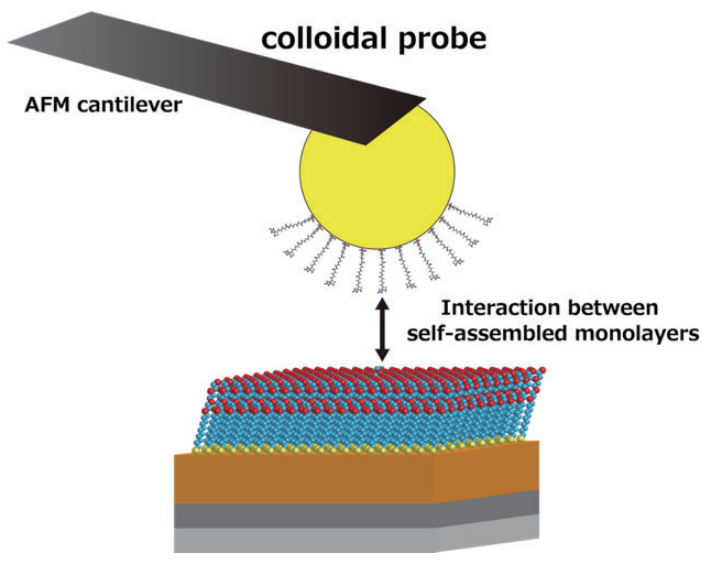

(b)

Fig. 1. (Color online) (a) Scanning electron microscopy image of a colloidal probe. A silica colloid is immobilized onto a cantilever. (b) Schematic illustration of a surface force measurement using a colloidal probe. The surfaces of the probe and substrate can be freely chosen by employing SAMs or other surface modifiers. The measurement environment (air, liquid, or vacuum) can also be flexibly chosen.

The contribution of water-mediated forces to protein or cell resistance has been speculated for a long time. ${ }^{(15-19)}$ In particular, the self-assembled monolayers (SAMs) of oligo(ethylene glycol)- or zwitterion (e.g., phosphorylcholine, carboxy- and sulfuric betaines)-terminated alkanethiols are highly protein- and cell-resistant and often used to suppress nonspecific adsorption in biosensing. However, because of the lack of experimental evidence for the force responsible for the protein and cell resistance, the underlying mechanism has not been clarified. Hayashi and coworkers first reported that the interfacial water near bioinert SAMs acts as a physical barrier to prevent the adsorption of proteins and the adhesion of cells by surface force measurements by AFM employing colloidal probes [the experimental configuration is shown in Fig. 1(b)]. ${ }^{(20-24)}$ They reported that the working distance of water-mediated repulsion greatly exceeds that of the DLVO force. Recently, Araki et al. also visualized the water barrier using frequency-modulation AFM near a zwitterionic bioinert SAM, supporting the results of Hayashi's group. ${ }^{(25)}$

In biological systems, biomolecules (e.g., proteins, lipids, DNA, and polysaccharides) specifically interact with their target molecules while suppressing non-specific interactions with other molecules. In many cases, the physicochemical origin of a specific biological interaction can be explained by considering the molecular structure of the binding sites of the ligands and receptors. However, the mechanism underlying the suppression of non-specific interactions cannot be explained easily. In fact, protein molecules in body fluids maintain their colloidal stability without forming aggregates. Valle-Delgado et al. reported a non-DLVO force acting between ferritin molecules, which are spherical cage-type proteins. ${ }^{(26)}$ This finding is supported by the results of static light scattering, which revealed a stable water layer covering the ferritin surface. ${ }^{(27)}$

Theoretical works using molecular dynamics also support the stable formation of a hydration layer around protein molecules and indicate that the dynamics of water molecules is about $10^{2}$ 
to $10^{3}$ times slower than that of bulk water in terms of rotational and translational structural relaxation. ${ }^{(28)}$ Collecting these findings, many researchers speculate that this water layer acts as a protective barrier to suppress non-specific interactions with non-target molecules.

White et al. performed a statistical analysis on the population of amino acids on the surfaces of 1162 human proteins and discovered that the residues of lysine and glutamic acid have the highest and second-highest populations, respectively. ${ }^{(29)}$ Interestingly, they also found that these residues often exist as zwitterionic pairs in the protein surfaces. ${ }^{(30)}$ On the basis of this finding, they constructed SAMs of peptides including the repeating units of the zwitterionic pairs of amino acid residues. They found that the SAMs exhibited high bioinertness (protein resistance). Chang et al. performed surface force analysis of peptide-based SAMs, reported that a stable water layer exists near the bioinert peptide SAMs, and proved that interfacial water is responsible for the bioinertness of peptide SAMs. ${ }^{(31)}$

DNA molecules are also essential biomolecules. Fujita et al. reported that the interaction between DNA helices strongly depends on the complementarity of terminal base pairs. When the terminal base pairs are complementary, the DNA molecules aggregate. On the other hand, when the terminal base pairs have a mismatch, the DNA molecules repel each other. ${ }^{(32)}$ Kanayama et al. investigated the force acting between the monolayers of DNA layers. They observed hydrophobic attraction and water-induced repulsion for complementary DNA and mismatched DNA layers, respectively, indicating that the water structure and behavior strongly depend on the complementarity of terminal base pairs. ${ }^{(33,34)}$ The above reports imply that biomolecules smartly use the interfacial water around them to function more than we expected.

\section{Single-molecule Force Spectroscopy and Force Spectroscopic Mapping}

\subsection{Single-molecule force spectroscopy}

AFM is also capable of measuring intra- or intermolecular forces at a single-molecule level. In single-molecule force spectroscopy, roughly speaking, there are two kinds of measurements. One is the unfolding of a single polymeric molecule by AFM. The terminals of the molecules are attached to the tip and substrate. Then, the distance between the tip and the sample's surface is increased while monitoring the force acting between them. In the cases of unfolding protein molecules, the distribution of the local mechanical stability of subunits in the molecule can be elucidated from the force-distance curve. There are reports on the unfolding and refolding processes of protein molecules using single-molecule force spectroscopy, demonstrating that force measurements can also be used to analyze the dynamics of biomolecule self-structuring and internal mechanical stability. ${ }^{(35,36)}$ In the cases of single-chain synthetic polymers, the stretching behavior strongly depends on the solvent, indicating that the picture of polymers dissolving in solvents can be discussed through single-molecule mechanics. ${ }^{(37,38)}$

The other kind of measurement is force measurements of the interactions between ligands and their receptors, which are immobilized on the probe and substrate. Then, the force required to rupture the bond is measured. Of course, the magnitude of the rupture force reflects the strength of the bond. The critical finding discovered by Evans et al. is that the rupture force 
critically depends on the loading speed during the rupturing, i.e., the faster the pulling, the larger the rupture force. From the rupture force profile plotted as a function of loading speed, we can evaluate the number and positions of the potential barriers in the bond and the bond's natural lifetime. This method (so-called dynamic force spectroscopy) unveiled a detailed picture of the potential landscape of the streptavidin (SA)-biotin bond, which is the strongest non-covalent bond in nature. ${ }^{(39)}$ Evans et al. reported that at least two potential barriers exist between fully bound and unbound states, and several other works also support this finding. ${ }^{(40,41)}$

The work by Evans et al. was performed by cell-membrane force spectroscopy, in which the force was evaluated by the deformation of red blood cells. Later, the SA-biotin bond was investigated by single-molecule force spectroscopy using $\mathrm{AFM}^{(40,42)}$ and optical tweezers. ${ }^{(43)}$ Interestingly, the results obtained by the different methods exhibited dissimilar features including the rupture force and the number of potential barriers. ${ }^{(44)}$

Single-molecule force spectroscopic measurements through monitoring the force with a high temporal resolution by Mondarte et al. revealed that there are at least six intermediate states between bound and unbound states. This finding is in agreement with the results of computer simulations. We assume that the reaction pathway along the reaction coordinate may depend on the loading speed because of the structural flexibility of SA. ${ }^{(45)}$ To explain this discrepancy clearly, an analysis to acquire a detailed picture of the unbinding process using molecular simulations is underway.

\subsection{Force spectroscopic mapping}

By mapping the forces acting between a tip and surface across a scan area, we can acquire physicochemical properties of samples that cannot be obtained by standard topographic imaging. Even with unmodified probes, we can image the chemical contrast of surfaces. For example, Suthiwanich et al. performed a chemical mapping of nanophase-separated block copolymers using a bare silicon nitride tip by measuring the adhesion force in water. ${ }^{(46)}$

Chemical modification of a tip enables us to visualize targeted materials or molecules. Arai et al. modified a tip surface with a material-binding peptide (a Ti-binding peptide) and succeeded in the chemical mapping of surfaces with nanoscale resolution. ${ }^{(47)}$ Müller et al. visualized the local distribution of receptor molecules on a cell membrane by immobilizing ligand molecules on a probe. ${ }^{(48)}$ In particular, they distinguished two different receptor proteins from their rupture lengths by employing two linkers with different molecular lengths, demonstrating the "multicolor" imaging of molecular recognition. By attaching appropriate molecules to the tip, we can expand the range of applications of this technique.

\subsection{Chemical modification of tips and substrates for single-molecule force spectroscopy and force spectroscopic mapping}

This section describes the details of probe modification for single-molecule force spectroscopic and force spectral mapping measurements. The chemical modification of the tip and substrate to achieve the dominant occurrence of single-molecule events is an essential 
part of the measurements. ${ }^{(49)}$ SAMs are often employed as templates for the immobilization of biomolecules on the tip and substrates (Fig. 2). Depending on the material of the tip, we select molecules with appropriate anchoring groups such as thiol, silane, phosphate, and isocyanide groups. The preparation (immersion, vapor deposition, etc.) of these SAMs depends on the combination of the anchoring groups and substrates. ${ }^{(50-57)}$

To enhance the probability of the formation of molecular bonds between ligands and receptors, a flexible polymer chain [usually poly(ethylene glycol)] is inserted between the SAM and ligands or receptors. The SAM must comprise two kinds of components: molecules with reactive terminal groups bound to biomolecules or ligands, and molecules with bioinert terminal groups to prevent non-specific interactions. By optimizing the ratio between them, singlemolecule events can be detected predominantly in force spectroscopic measurements. For the choice of the chemical reaction for the immobilization, we refer readers to Ref. 58. ${ }^{(58)}$

\section{Other SPM-related Techniques in Nano-bioscience}

We can combine AFM with other techniques. If AFM allows optical access from above, below, or the side of the sample, it can be integrated with optical microscopy. For example, a fluorescence (FL) microscope is an essential tool in cell biology. FL microscopes can visualize protein molecules of interest to the researcher, and AFM can visualize the detailed geometry of cells and the local structure of cell membranes at the nanoscale.

AFM combined with Raman microscopy is also a powerful technique for characterizing biomaterials and biomolecules at multiple scales. Although there is a gap in the spatial

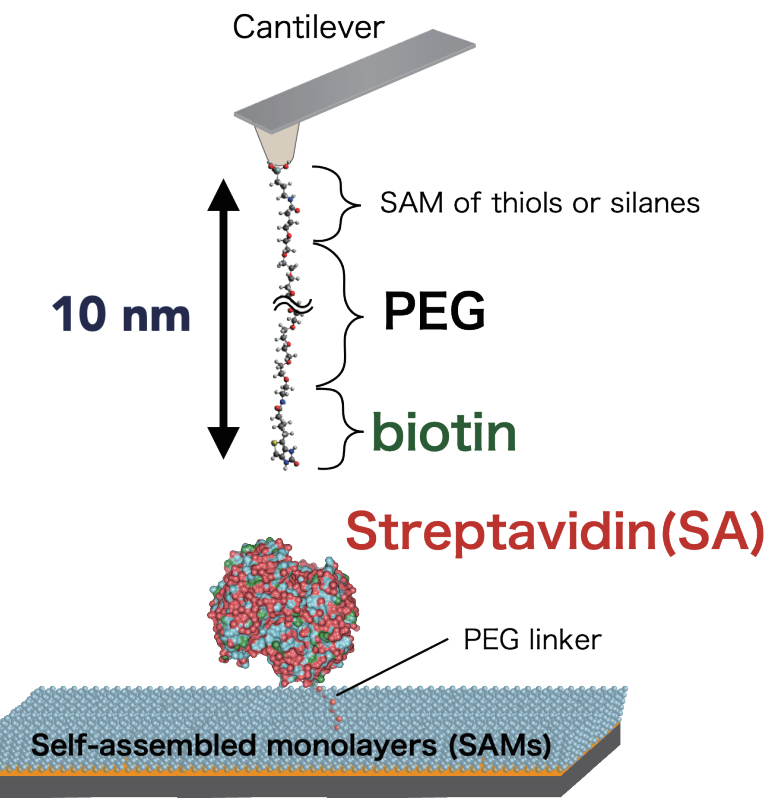

Fig. 2. (Color online) Typical configuration for single-molecule force spectroscopy for ligand-receptor systems. Here, the SA-biotin system is presented. 
resolution between Raman microscopy and AFM, they provide topographical and chemical information. Therefore, commercial systems combining Raman microscopy and AFM have been released. Tip-enhanced Raman spectroscopy (TERS) was invented in the 2000s to achieve Raman spectroscopy with high spatial resolution. ${ }^{(59)}$ In this technique, AFM probes are covered with a noble metal (usually silver or gold), and laser light is focused on the tip apex. Under the irradiation, the plasmonic resonance between the laser light and the collective motion of free electrons (plasmon) occurs if their frequencies are close to each other. AFM-based TERS accomplished nanometer-scale resolution, with which defects in carbon nanotubes were visualized by spectral mapping. ${ }^{(60,61)}$

Unfortunately, TERS has an inevitable local heating problem at the tip apex due to Joule heating originating from the plasmonically induced motion of electrons. Therefore, there is a potential risk of heating damage to biomolecules or cells. Mochizuki et al. and Balois et al. previously evaluated the temperature at a tip apex both optically and chemically. ${ }^{(62-64)}$ Mochizuki et al. also reported that the oscillation of the cantilever promotes the dissipation of heat into the media. Therefore, we firmly believe that TERS can be used to characterize biological samples with the optimization of experimental conditions.

\section{Conclusions}

In this review, we introduced the application of AFM in the field of nano-bioscience. In particular, we described works that employed AFM as a nanosized toolbox for tasks beyond simple imaging. New applications of AFM techniques in nano-bioscience and technology have been continuously reported, which include integration with microfluidics, ${ }^{(65,66)}$ and a mechanical sensor to evaluate the mechanical properties of living cells. ${ }^{(67,68)}$ There is plenty of room for new applications of AFM in this field by combining tip modification and conjugation with other analytical techniques, where the only limits are our originality and ingenuity.

\section{Acknowledgments}

We acknowledge the administration of Ms. Kazue Taki for the preparation of this review.

\section{References}

1 G. Binning, H. Rohrer, C. Gerber, and E. Weibel: Phys. Rev. Lett. 49 (1982) 57. https://oi.org/10.1103/ PhysRevLett.49.57

2 G. K. Binnig: Phys. Scr. T19A (1987) 53. https://doi.org/10.1088/0031-8949/1987/T19a/008

3 D. J. Muller and Y. F. Dufrene: Nat. Nanotechnol. 3 (2008) 261. https://doi.org/10.1038/nnano.2008.100

4 S. Vahabi, B. Nazemi Salman, and A. Javanmard: Iran J. Med. Sci. 38 (2013) 76.

5 E. S. Gyorvary, O. Stein, D. Pum, and U. B. Sleytr: J. Microsc. 212 (2003) 300. https://doi.org/10.1111/j.13652818.2003.01270.x

6 H. Lin, D. O. Clegg, and R. Lal: Biochemistry 38 (1999) 9956. https://doi.org/10.1021/bi990800q

7 J. N. Israelachvili: Intermolecular and Surface Forces (Elsevier, 2011) 3rd ed.

8 H. J. Butt, B. Cappella, and M. Kappl: Surf. Sci. Rep. 59 (2005) 1. https://doi.org/10.1016/j.surfrep.2005.08.003

9 J. Parker: Langmuir 8 (1992) 551.

10 K. E. Bremmell, L. Britcher, and H. J. Griesser: Colloids Surf. B Biointerfaces 106 (2013) 102. https://doi. org/10.1016/j.colsurfb.2013.01.052 
11 K. E. Bremmell, P. Kingshott, Z. Ademovic, B. Winther-Jensen, and H. J. Griesser: Langmuir 22 (2006) 313. https://doi.org/10.1021/la052143a

12 M. Leivers, J. M. Seddon, M. Declercq, E. Robles, and P. Luckham: Langmuir 35 (2019) 729. https://doi. org/10.1021/acs.langmuir.8b03555

13 A. Striolo: Adsorpt. Sci. Technol. 29 (2011) 211. https://doi.org/10.1260/0263-6174.29.3.211

14 E. A. Vogler: Adv. Colloid Interface Sci. 74 (1998) 69. https://doi.org/10.1016/S0001-8686(97)00040-7

15 T. Hayashi and M. Hara: Current Phys. Chem. 1 (2011) 90.

16 A. J. Pertsin, T. Hayashi, and M. Grunze: Phys. Chem. Chem. Phys. 3 (2001) 1598. https://doi.org/10.1039/ b100097g

17 T. Hayashi, A. J. Pertsin, and M. Grunze: J. Chem. Phys. 117 (2002) 6271. https://doi.org/10.1063/1.1504436

18 A. J. Pertsin, T. Hayashi, and M. Grunze: J. Phys. Chem. B 106 (2002) 12274. https://doi.org/10.1021/jp0257471

19 D. Schwendel, T. Hayashi, R. Dahint, A. Pertsin, M. Grunze, R. Steitz, and F. Schreiber: Langmuir 19 (2003) 2284. https://doi.org/10.1021/la026716k

20 T. Hayashi, Y. Tanaka, Y. Koide, M. Tanaka, and M. Hara: Phys. Chem. Chem. Phys. 14 (2012) 10196. https:// doi.org/10.1039/c2cp41236e

21 T. Sekine, Y. Tanaka, C. Sato, M. Tanaka, and T. Hayashi: Langmuir 31 (2015) 7100. https://doi.org/10.1021/ acs.langmuir.5b01216

22 T. Sekine, S. Asatyas, C. Sato, S. Morita, M. Tanaka, and T. Hayashi: J. Biomater. Sci. Polym. Ed. 28 (2017) 1231. https://doi.org/10.1080/09205063.2017.1303120

23 M. Tanaka, S. Morita, and T. Hayashi: Colloids Surf. B (2020). https://doi.org/10.1016/j.colsurfb.2020.111449

24 M. Tanaka, T. Hayashi, and S. Morita: Polym. J. (Tokyo, Jpn.) 45 (2013) 701. https://doi.org/10.1038/ pj.2012.229

25 Y. Araki, T. Sekine, R. Chang, T. Hayashi, and H. Onishi: RSC Adv. 8 (2018) 24660. https://doi.org/10.1039/ c8ra03569e

26 J. J. Valle-Delgado, J. A. Molina-Bolivar, F. Galisteo-Gonzalez, M. J. Galvez-Ruiz, A. Feiler, and M. W. Rutland: Langmuir 21 (2005) 9544. https://doi.org/10.1021/la050825s

27 D. N. Petsev and P. G. Vekilov: Phys. Rev. Lett. 84 (2000) 1339. https://doi.org/10.1103/PhysRevLett.84.1339

28 T. Wohlfromm and M. Vogel: J. Chem. Phys. 150 (2019) 245101. https://doi.org/10.1063/1.5097777

29 A. D. White, A. K. Nowinski, W. J. Huang, A. J. Keefe, F. Sun, and S. Y. Jiang: Chem. Sci. 3 (2012) 3488. https://doi.org/10.1039/c2sc21135a

30 A. K. Nowinski, F. Sun, A. D. White, A. J. Keefe, and S. Jiang: J. Am. Chem. Soc. 134 (2012) 6000. https://doi. org $/ 10.1021 / \mathrm{ja} 3006868$

31 R. Chang, S. Asatyas, G. Lkhamsuren, M. Hirohara, E. A. Q. Mondarte, K. Suthiwanich, T. Sekine, and T. Hayashi: Polym. J. (Tokyo, Jpn.) 50 (2018) 563. https://doi.org/10.1038/s41428-018-0075-1

32 M. Fujita, Y. Katafuchi, K. Ito, N. Kanayama, T. Takarada, and M. Maeda: J. Colloid Interface Sci. 368 (2012) 629. https://doi.org/10.1016/j.jcis.2011.11.021

33 N. Kanayama, T. Sekine, K. Ozasa, S. Kishi, T. Nyu, T. Hayashi, and M. Maeda: Langmuir 32 (2016) 13296. https://doi.org/10.1021/acs.langmuir.6b03470

34 T. Sekine, N. Kanayama, K. Ozasa, T. Nyu, T. Hayashi, and M. Maeda: Langmuir 34 (2018) 15078. https://doi. org/10.1021/acs.langmuir.8b02224

35 D. R. Jacobson, L. Uyetake, and T. T. Perkins: Biophys. J. 118 (2020) 667. https://doi.org/10.1016/j.bpj.2019.12.003

36 T. Bornschlogl and M. Rief: Methods Mol. Biol. 783 (2011) 233. https://doi.org/10.1007/978-1-61779-282-3 13

37 M. Rief, F. Oesterhelt, B. Heymann, and H. E. Gaub: Science 275 (1997) 1295. https://doi.org/10.1126/ science. 275.5304 .1295

38 F. Oesterhelt, M. Rief, and H. E. Gaub: New J. Phys. 1 (1999). https://doi.org/10.1088/1367-2630/1/1/006

39 R. Merkel, P. Nassoy, A. Leung, K. Ritchie, and E. Evans: Nature 397 (1999) 50. https://doi.org/10.1038/16219

40 A. Taninaka, O. Takeuchi, and H. Shigekawa: Int. J. Mol. Sci. 11 (2010) 2134. https://doi.org/10.3390/ ijms11052134

41 A. Taninaka, Y. Hirano, O. Takeuchi, and H. Shigekawa: Int. J. Mol. Sci. 13 (2012) 453. https://doi.org/10.3390/ ijms13010453

42 A. Taninaka, O. Takeuchi, and H. Shigekawa: Phys. Chem. Chem. Phys. 12 (2010) 12578. https://doi. org $/ 10.1039 / \mathrm{c} 0 \mathrm{cp} 00259 \mathrm{c}$

43 T. Ota, T. Sugiura, and S. Kawata: Appl. Phys. Lett. 87 (2005) 043901. https://doi.org/10.1063/1.1999855

44 F. Pincet and J. Husson: Biophys. J. 89 (2005) 4374. https://doi.org/10.1529/biophysj.105.067769

45 E. A. Mondarte, T. Maekawa, T. Nyu, H. Tahara, G. Lkhamsuren, and T. Hayashi: RSC Adv. 9 (2019) 22705. https://doi.org/10.1039/c9ra04106k

46 K. Suthiwanich, Y. Hiraguchi, T. Nyu, E. A. Q. Mondarte, M. Takai, and T. Hayashi: Chem. Lett. 49 (2020) 641. 
47 Y. Arai, K. Okabe, H. Sekiguchi, T. Hayashi, and M. Hara: Langmuir 27 (2011) 2478. https://doi.org/10.1021/ la104178h

48 M. Pfreundschuh, D. Alsteens, R. Wieneke, C. Zhang, S. R. Coughlin, R. Tampe, B. K. Kobilka, and D. J. Muller: Nat. Commun. 6 (2015) 8857. https://doi.org/10.1038/ncomms9857

49 H. Tahara, T. Nyu, E. A. Q. Mondarte, T. Maekawa, and T. Hayashi: Adv. Mater. Phys. Chem. 8 (2018) 217. https://doi.org/10.4236/ampc.2018.85014

50 G. Lkhamsuren, M. Mochizuki, K. Suthiwanich, and T. Hayashi: Thin Solid Films 636 (2017) 478. https://doi. org/10.1016/j.tsf.2017.06.046

51 J. C. Love, L. A. Estroff, J. K. Kriebel, R. G. Nuzzo, and G. M. Whitesides: Chem. Rev. 105 (2005) 1103. https://doi.org/10.1021/cr0300789

52 A. Tsunoi, G. Lkhamsuren, E. A. Q. Mondarte, S. Asatyas, M. Oguchi, J. Noh, and T. Hayashi: J. Phys. Chem. C 123 (2019) 13681. https://doi.org/10.1021/acs.jpcc.9b02256

53 T. Hayashi, K. Wakamatsu, E. Ito, and M. Hara: J. Phys. Chem. C 113 (2009) 18795. https://doi.org/10.1021/ jp906494u

54 C. Kodama, T. Hayashi, and H. Nozoye: Appl. Surf. Sci. 169-170 (2001) 264. https://doi.org/10.1016/s01694332(00)00664-4

55 T. Hayashi, Y. Morikawa, and H. Nozoye: J. Chem. Phys. 114 (2001) 7615. https://doi.org/10.1063/1.1360245

56 T. Hayashi, C. Kodama, and H. Nozoye: Appl. Surf. Sci. 169 (2001) 100. https://doi.org/10.1016/S01694332(00)00647-4

57 T. Hayashi, A. Fricke, K. Katsura, C. Kodama, and H. Nozoye: Surf. Sci. 427-428 (1999) 393. https://doi. org/10.1016/S0039-6028(99)00308-8

58 G. T. Hermanson: Bioconjugate Techniques (Academic Press, Cambridge, 2013) 3rd ed.

59 J. J. Wang, D. A. Smith, D. N. Batchelder, Y. Saito, J. Kirkham, C. Robinson, K. Baldwin, G. Li, and B. Bennett: J. Microsc. 210 (2003) 330. https://doi.org/10.1046/j.1365-2818.2003.01166.x

60 N. Kumar, B. M. Weckhuysen, A. J. Wain, and A. J. Pollard: Nat. Protoc. 14 (2019) 1169. https://doi. org/10.1038/s41596-019-0132-Z

61 M. Oguchi, M. Mochizuki, T. Yano, M. Hara, and T. Hayashi: Chem. Lett. 43 (2014) 808. https://doi. org/10.1246/cl.140093

62 M. V. Balois, N. Hayazawa, F. C. Catalan, S. Kawata, T. A. Yano, and T. Hayashi: Anal. Bioanal. Chem. 407 (2015) 8205. https://doi.org/10.1007/s00216-015-8866-0

63 M. Mochizuki, G. Lkhamsuren, K. Suthiwanich, E. A. Mondarte, T. A. Yano, M. Hara, and T. Hayashi: Nanoscale 9 (2017) 10715. https://doi.org/10.1039/c7nr02398g

64 M. Mochizuki, S. Asatyas, K. Suthiwanich, and T. Hayashi: Chem. Lett. 45 (2016) 1207. https://doi. org/10.1246/cl.160572

65 M. Deliorman, F. K. Janahi, P. Sukumar, A. Glia, R. Alnemari, S. Fadl, W. Q. Chen, and M. A. Qasaimeh: Microsyst. Nanoeng. 6 (2020). https://doi.org/10.1038/s41378-020-0131-9

66 T. Maekawa, H. Chin, T. Nyu, T. N. Sut, A. R. Ferhan, T. Hayashi, and N. J. Cho: Phys. Chem. Chem. Phys. 21 (2019) 16686. https://doi.org/10.1039/c9cp02085c

67 Y. M. Efremov, T. Okajima, and A. Raman: Soft Matter 16 (2020) 64. https://doi.org/10.1039/c9sm01020c

68 M. Lekka, D. Gil, K. Pogoda, J. Dulinska-Litewka, R. Jach, J. Gostek, O. Klymenko, S. Prauzner-Bechcicki, Z. Stachura, J. Wiltowska-Zuber, K. Okon, and P. Laidler: Arch. Biochem. Biophys. 518 (2012) 151. https://doi. org/10.1016/j.abb.2011.12.013

\section{About the Authors}

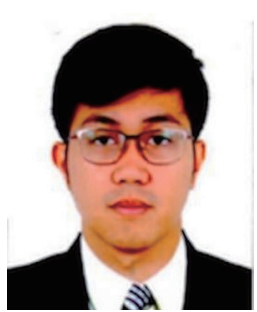

Evan Angelo Quimada Mondarte received his B.S. and M.Sc. degrees from the University of the Philippines - Diliman, Philippines, in 2011 and 2015, respectively, and his M.Eng. degree from Tokyo Institute of Technology, Japan, in 2018. His research interests are in surface science, bioengineering, and materials science. (mondarte.e.aa@m.titech.ac.jp) 


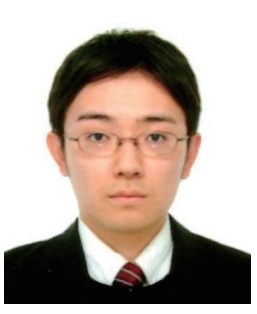

Hiroyuki Tahara received his B.S. degree from the University of ElectroCommunications, Japan, in 2017 and his M.S. degree from Tokyo Institute of Technology, Japan, in 2019. His research interests are in surface science, bioengineering, and materials informatics. (tahara.h.ab@m.titech.ac.jp)

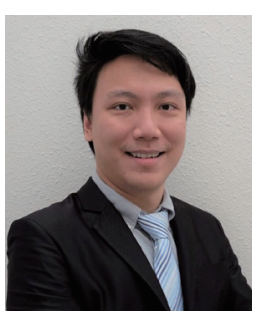

Kasinan Suthiwanich received his B.Eng. degree from Chulalongkorn University, Thailand, in 2015 and his M.Eng. and D.Sc. degrees from Tokyo Institute of Technology, Japan, in 2017 and 2020, respectively. His research backgrounds include biointerface science, polymer nanomechanics, and atomic force microscopy. From 2017 to 2018, he was a visiting graduate researcher at the University of California Los Angeles. He is currently one of the postdoctoral staff at RIKEN. (suthiwanich.k.aa@m.titech.ac.jp)

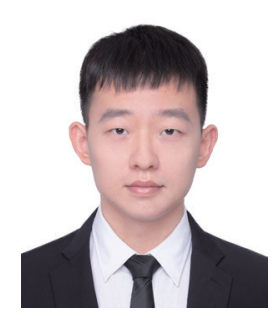

Subin Song received his B.S. degree from Anhui University of Technology, China, in 2016 and his M.S. degree from the University of Science and Technology of China in 2019. His research interests are in materials engineering, surface science, and bioengineering. (song.s.ad@m.titech.ac.jp)

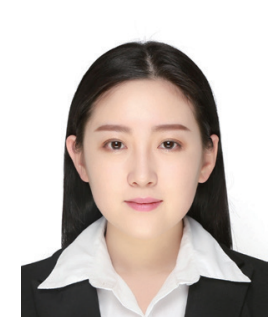

Fan Wang received her B.S. degree from Inner Mongolia Normal University, China, in 2018. Her research interests are in surface science, materials science, and bioengineering. (wang.f.ab@m.titech.ac.jp)

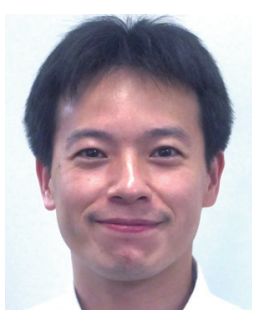

Tomohiro Hayashi received his Ph.D. degree in 2003 from Ruprecht-KarlsUniversität Heidelberg. He joined Tokyo Institute of Technology in 2003 as a postdoc and was promoted to an associate professor in 2010. His specialist areas are surface and interface science, scanning probe microscopy, materials informatics, and computer simulations. He has been awarded 11 academic prizes, including the Asahi Kasei award of the Society of Polymer Science, Japan (2011). A summary of his activities is given at http://lab.spm.jp/. (tomo@mac.titech.ac.jp) 